\title{
Contextual Influences on Phonetic Identification in Aphasia: The Effects of Speaking Rate and Semantic Bias
}

\author{
Shari R. Baum \\ School of Communication Sciences and Disorders, McGill University, Montreal, Quebec, Canada
}

Published online February 15, 2001

\begin{abstract}
Two experiments examined the influence of context on stop-consonant voicing identification in fluent and nonfluent aphasic patients and normal controls. Listeners were required to label the initial stop in a target word varying along a voice onset time (VOT) continuum as either voiced or voiceless $([\mathrm{b}] /[\mathrm{p}]$ or $[\mathrm{d}] /[\mathrm{t}])$. Target stimuli were presented in sentence contexts in which the rate of speech of the sentence context (Experiment 1) or the semantic bias of the context (Experiment 2) was manipulated. The results revealed that all subject groups were sensitive to the contextual influences, although the extent of the context effects varied somewhat across groups and across experiments. In addition, a number of patients in both the fluent and nonfluent aphasic groups could not consistently identify even endpoint stimuli, confirming phonetic categorization impairments previously shown in such individuals. Results are discussed with respect to the potential reliance by aphasic patients on higher level context to compensate for phonetic perception deficits. ๑ 2001 Academic Press
\end{abstract}

Impairments in the ability to identify phonetic segments-particularly consonantal segments - are often observed in aphasia (Gow \& Caplan, 1996) and may account, in part, for deficits in word recognition in left-hemisphere-damaged patients (Blumstein, 1991). Recent studies have also shown that higher level contextual influences may help patients to compensate for deficits in phonetic perception (e.g., Caplan \& Aydelott-Utman, 1994). Investigations of normal speech perception have demonstrated numerous context effects in phonetic identification, supporting an interactive speech processing system (e.g., Borsky, Tuller, \& Shapiro, 1998; Connine, 1987; Elman \& McClelland, 1986; Fox, 1984; Ganong, 1980; Miller, Dexter, \& Pickard, 1984; Pitt \& Samuel, 1993; Samuel, 1981; Warren, 1970). In particular, such contextual influences tend to emerge most prominently when the acoustic input is less than natural or degraded (Burton, Baum, \& Blumstein, 1989; McQueen, 1991). If impairments in phonetic perception in aphasia are thought of as providing the patients with degraded input, one might predict an increased influence of higher level information in braindamaged patients as compared to normals.

Building on the findings in the normal speech perception literature, Blumstein and colleagues (1994) examined whether nonfluent (Broca's) aphasics and fluent (Wernicke's and Conduction) aphasics exhibited lexical influences in a phonetic categori-

This research was supported by a grant from the Medical Research Council of Canada. Thanks are due to Jeff Boyczuk for comments on an earlier draft.

Address correspondence and reprint requests to Shari R. Baum, School of Communication Sciences and Disorders, McGill University, 1266 Pine Avenue West, Montreal, QC H3G 1A8 Canada. E-mail: shari.baum@mcgill.ca. 
zation task. Subjects were tested on identification of edited natural stimuli varying along two $[\mathrm{d}]-[\mathrm{t}]$ voice onset time (VOT) continua (see Burton et al., 1989 for a detailed description of stimulus creation). In one continuum, the voiced endpoint formed a real word in English ("duke") and the voiceless endpoint formed a nonword ("tuke"). In the other continuum, only the voiceless endpoint formed a real word ("doot"/"toot"). Previous studies have shown that the lexical status of a stimulus influences phonetic identification in such tasks, yielding a higher percentage of voiced responses when the voiced endpoint forms a real word (and conversely, more voiceless responses when the voiceless endpoint forms a real word) - the so-called lexical effect (e.g., Fox, 1984; Ganong, 1980).

Blumstein et al. (1994) found that nonfluent aphasic patients displayed a larger than normal lexical effect, whereas fluent aphasic patients exhibited no lexical effect. The authors interpreted their findings as indicating that nonfluent aphasic patients may rely on lexical information as a strategy to compensate for impaired phonetic perception; fluent aphasics, in contrast, were unable to utilize the lexical information due, perhaps, to more general lexical processing impairments (Blumstein et al., 1994).

In an effort to both replicate and extend Blumstein et al.'s (1994) results, Boyczuk and Baum (1999) tested groups of fluent and nonfluent aphasic patients and normal controls on two sets of VOT continua. One of the sets of continua utilized word and nonword endpoints (as in the lexical effects studies described above), but controlled the stimuli for neighborhood density (Luce, 1987) - a factor that has been suggested as a possible confounding variable in lexical effect studies (Newman, Sawusch, \& Luce, 1997).

Neighborhood density refers to the number and frequency of phonologically related words presumably activated upon hearing a stimulus (Luce, 1987) and reflects an additional source of lexical information that may affect phonetic identification. Following Newman et al. (1997), the second set of VOT continua in Boyczuk and Baum's (1999) study utilized only nonword endpoints which differed in terms of their neighborhood density values. The results of these experiments revealed that all subject groups showed an influence of both lexical status and neighborhood density in rendering phonetic identification decisions. Although the findings for the aphasic patients with regard to the influence of lexical status are not in keeping with those reported by Blumstein et al. (1994), the inconsistency may be explained by the specific stimuli used and the manipulation of neighborhood density in Boyczuk and Baum's (1999) study. In order to ensure that neighborhood density would not contribute to a lexical effect, Boyczuk and Baum (1999) were required to select stimuli for which the potential influence of neighborhood density conflicted with that of lexical status; that is, the nonword endpoints had higher neigborhood density values, possibly working against the bias of lexical status. The two conflicting top-down influences may account for the absence of evidence supporting an increased reliance on higher level information in the nonfluent aphasic patients (Blumstein et al., 1994). In fact, some indication of such an increased dependence on contextual information was found in the experiment exploring neighborhood density alone, with the aphasic patients displaying more of a tendency toward a neighborhood density effect than normal controls (Boyczuk \& Baum, 1999).

The results for the fluent aphasic patients were equally unexpected, given the absence of a lexical influence in Blumstein et al.'s (1994) earlier study. However, these findings may also be explained, in part, with reference to the specific stimuli utilized in the two studies. Pitt and Samuel (1993) have shown that there is a great deal of inconsistency in the emergence of a lexical effect in studies examining the $[\mathrm{d}]-[\mathrm{t}]$ contrast (i.e., the contrast employed by Blumstein et al., 1994). Lexical effects seem to emerge more consistently in studies examining the labial [b]-[p] 
contrast utilized in Boyczuk and Baum's (1999) investigation. Moreover, the fluent aphasic patients tested by Boyczuk and Baum exhibited the largest effect of neighborhood density in the nonword-nonword continua. This may be interpreted in keeping with the claim that fluent aphasic patients may overactivate lexical candidates (Blumstein, Milberg, \& Shrier, 1982; Leonard \& Baum, 1997; Milberg, Blumstein, \& Dworetzky, 1988), thereby increasing activation of lexical neighbors and resulting in an increased influence of neighborhood density on phonetic categorization abilities.

Although the results reported by Boyczuk and Baum (1999) yield some support for the hypothesis posited by Blumstein et al. (1994) concerning increased (perhaps strategic) reliance on higher level information in speech perception, the data leave many questions unresolved. The present study attempts to examine two other contextual influences which may help to clarify the speech processing abilities of both fluent and nonfluent aphasic patients: the influence of speaking rate and the influence of semantic context.

Investigations have shown that normal listeners' identification of ambiguous stimuli along a VOT continuum is modified by the speaking rate of a carrier phrase, such that a shorter VOT is required to perceive a voiceless stop in the context of a fast rate of speech relative to a slow rate of speech (Miller et al., 1984; see also Summerfield, 1981). Similarly, the semantic context in which a target word appears may serve to bias phonetic identification, shifting the category boundary away from the biased endpoint (i.e., yielding more responses consistent with the semantic bias) (Borsky et al., 1998; Connine, 1987; Connine, Blasko, \& Hall, 1991; Miller et al., 1984).

The objective of the present study was to further test the hypothesis that nonfluent aphasic patients rely more heavily than normals on higher level context to aid in phonetic identification (Blumstein et al., 1994; see also Boyczuk \& Baum, 1999) and to clarify the inconsistent results for fluent aphasic patients gathered to date. To this end, two experiments were designed to explore rate-of-speech effects and semantic context effects in the identification of voicing in initial stop consonants. For each experiment, VOT continua were constructed for two places of articulation (labial and alveolar) to ensure that the results would not be specific to a particular stimulus set (Pitt \& Samuel, 1993).

Although, like the lexical and neighborhood density effects studied in previous research, the influence of speaking rate and semantic context represent higher level contextual influences on speech perception, each derives from a different level of processing, perhaps reflecting automatic and strategic processing to different degrees [particularly in modular models (e.g., Fodor, 1983) of language processing (Miller et al., 1984; but cf. Borsky et al., 1997)]. We might therefore predict somewhat different patterns of performance for the different groups of aphasic patients on the two experimental tasks. That is, the influence of speaking rate, although a function of the sentence context, represents a change in the acoustic-phonetic structure of the input and may thus reflect a more direct unavoidable (i.e., automatic) adjustment in speech processing (Miller et al., 1984). Lexical effects, in contrast, have been shown to be subject to influence by changes in task demands and stimulus quality (Burton et al., 1989; Fox, 1984) and may thus reflect postperceptual (postphonetic) processing. Finally, the influence of sentential semantic context is clearly the furthest removed ${ }^{1}$ from the process of phonetic identification, as it requires not only word recognition and access to lexical knowledge, but also the build-up and integration of that informa-

${ }^{1}$ This is, of course, less true in an interactive account of language processing (e.g., McClelland \& Elman, 1986), although presumably more local versus more global context would still yield somewhat different effects. 
tion across an entire sentence. Its emergence, too, is subject to the influence of varying task demands and is thus not likely to be automatic (Miller et al., 1984; see also Borsky et al., 1998; Connine, 1987 for different interpretations).

With respect to the performance of aphasic patients, a number of predictions may be made. It is well known that nonfluent aphasic patients have difficulty processing the temporal parameters of speech (see, e.g., Baum, 1998; Baum, Pell, Leonard, \& Gordon, 1997); thus, they may be less susceptible to speaking-rate influences than normal subjects or fluent aphasic patients. As described earlier, it has also been suggested that nonfluent aphasic patients rely on contextual information to a greater extent than normal, which may yield a stronger effect of semantic context in this subject group. In contrast, fluent aphasic patients may be less susceptible to the influence of semantic context, given their impaired sentence comprehension abilities.

\section{METHODS}

\section{Subjects}

The subjects included groups of left-hemisphere-damaged (LHD) aphasic patients $(n=20)$ and age-matched normal controls $(n=12)$. The aphasic patients were classified into groups according to fluency (13 nonfluent and 7 fluent patients). All subjects were native speakers of English whose hearing was screened to be within normal limits. The normal control subjects had no history of neurological or speech-language disorders. Brain-damaged patients had all suffered a single unilateral cerebrovascular accident (confirmed by CT or MRI) and underwent a series of screening tests including sections of the Psycholinguistic Assessment of Language (PAL; (Caplan, 1992) and the Boston Diagnostic Aphasia Examination (BDAE; Goodglass \& Kaplan, 1983). Table 1 provides background information on the aphasic patients.

\section{Stimuli and Procedures-Rate of Speech (Experiment 1)}

The stimulus sentences were a subset of those utilized by Miller et al. (1984) and Connine (1987). Sentence frames consisted of the neutral sentence "She is not thinking of the __ spoken at two different rates of speech: fast and slow. Two target word continua were created, one ranging from "bath" to " path" and the other from "dent" to "tent." An adult male native speaker of English recorded multiple repetitions of the sentences (including the final target words) at three self-determined rates (fast, slow, and normal). All stimuli were digitized at a rate of $10 \mathrm{k}$ samples/s with a $4.5-\mathrm{kHz}$ low-pass filter and 12-bit quantization using the BLISS speech analysis system (Mertus, 1989). The normal rate sentences were utilized to extract and edit the target words. A single clear exemplar of a fast-rate and a slow-rate frame were chosen for each place of articulation. The selected fast-rate frame for the labial continuum was $980 \mathrm{~ms}$ in duration; the fast-rate frame for the alveolar continuum was $985 \mathrm{~ms}$. For the slow-rate frames, utterance lengths were $1666 \mathrm{~ms}$ for the labial continuum and $1631 \mathrm{~ms}$ for the alveolar continuum. These durations are consistent with those of stimuli in previous studies (e.g., Miller et al., 1984). Each frame was edited to remove the final target word and associated stop closure interval; the closure interval was excised to avoid providing cues other than VOT to the listeners.

To create the target word continua, one exemplar of "bath," " "path," " "dent," and "tent" was selected from the neutral, normal-rate sentences as representative of the majority of productions. Target words were delimited by the onset of the release burst for the initial stop and the end of noticeable frication (for the labial series) or final-consonant release (for the alveolar series). The voiced endpoint served as the base target-word for each continuum; target-word durations were $461 \mathrm{~ms}$ ("bath") and $397 \mathrm{~ms}$ ( "dent"). The VOTs of each endpoint were measured from the onset of the burst to the onset of periodicity associated with the vocalic transitions; these values are displayed in Table 2, along with the VOT values for each step on the continuum. For the "bath-path" series, an 11-step continuum was generated, whereas for the "dent-tent" series, a 14-step continuum was created. Stimuli along the continuum were created as follows (Blumstein et al., 1994; Boyczuk \& Baum, 1999; Burton et al., 1989). Successively longer segments of the voiceless stops beginning at the onset of the release burst were edited and inserted into (i.e., replaced) the voiced endpoint stimuli in which segments of equal length had been removed beginning at the stop onset. All cuts were made at zero crossings to avoid audible noise caused by the 


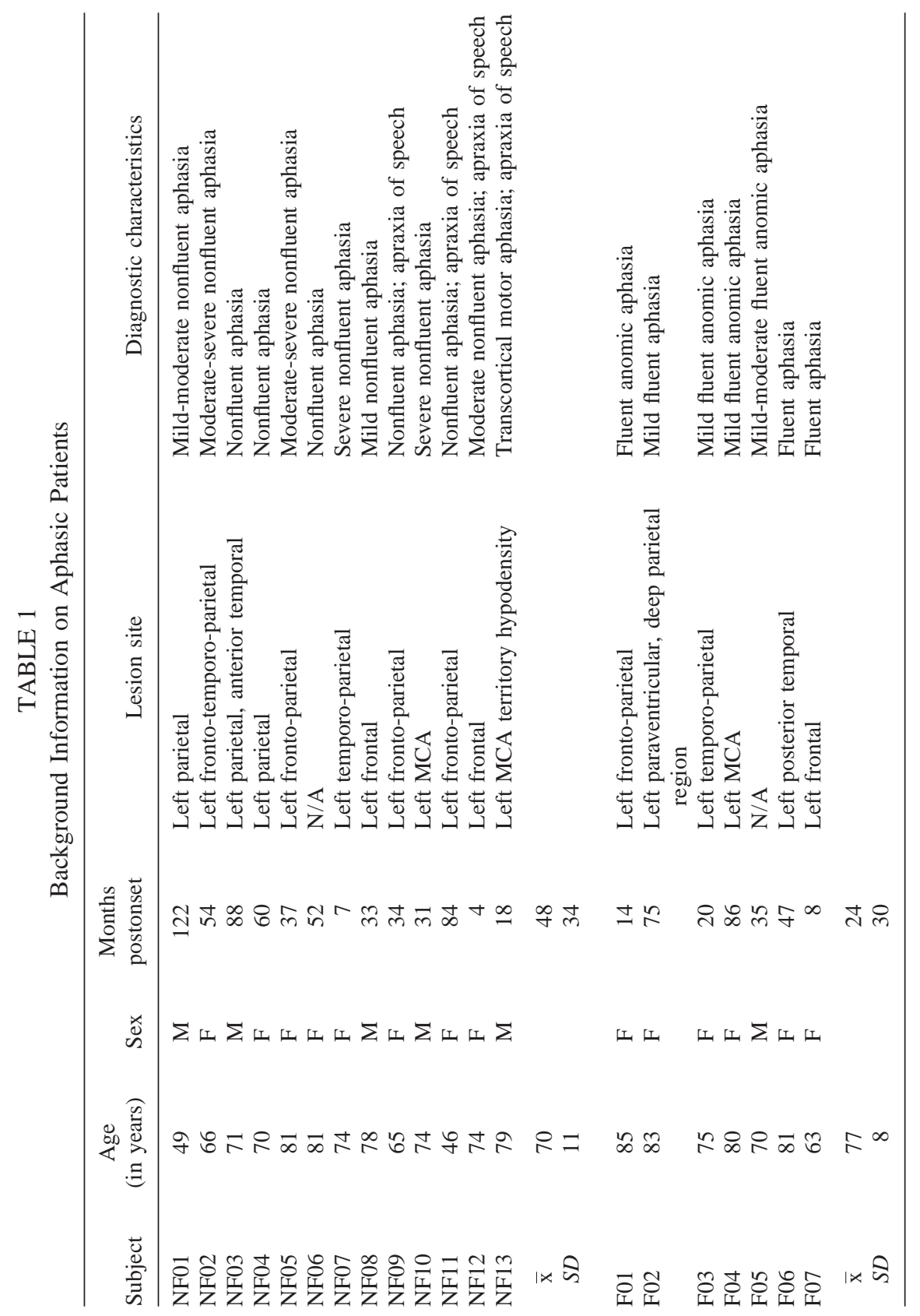




\section{TABLE 2}

Voice Onset Times and Step Sizes (in Milliseconds) for Stimuli along Labial and Alveolar Continua

\begin{tabular}{ccc}
\hline $\begin{array}{c}\text { Stimulus } \\
\text { number }\end{array}$ & $\begin{array}{c}\text { Voice } \\
\text { onset time } \\
\text { (in ms) }\end{array}$ & Step size \\
\hline $\begin{array}{c}\text { Bath/path } \\
0\end{array}$ & 11.5 & \\
1 & 16.1 & 4.6 \\
2 & 20.5 & 4.4 \\
3 & 24.9 & 4.4 \\
4 & 30.7 & 5.8 \\
5 & 34.2 & 3.5 \\
6 & 39.0 & 4.8 \\
7 & 44.2 & 5.2 \\
8 & 49.0 & 4.8 \\
9 & 54.4 & 5.4 \\
10 & 59.1 & 4.7 \\
Dent/tent & & \\
0 & 15.9 & - \\
1 & 19.7 & 3.8 \\
2 & 23.5 & 3.8 \\
3 & 27.5 & 4.0 \\
4 & 32.6 & 5.1 \\
5 & 36.7 & 4.1 \\
6 & 42.1 & 5.4 \\
7 & 45.5 & 3.4 \\
8 & 50.8 & 5.3 \\
9 & 55.6 & 4.8 \\
10 & 60.3 & 4.7 \\
11 & 65.2 & 4.9 \\
12 & 71.1 & 5.9 \\
13 & 75.8 & 4.7 \\
\hline & &
\end{tabular}

editing procedure. Step size was approximately $5 \mathrm{~ms}$, but varied from 3.4 to $5.8 \mathrm{~ms}$ across the two continua to accommodate the zero crossing criterion (see Table 2).

Each of the target stimuli (VOT continuum steps) was paired with the relevant fast-rate and slowrate frame using a 50-ms interstimulus interval (ISI), yielding a total of 22 stimuli for the [b]-[p] series and 28 stimuli for the [d]-[t] series. The stimuli were presented to listeners 10 times each in random order, with the order of the labial and alveolar stimulus sets counterbalanced across listeners within each group and across Experiments 1 and 2, in two separate sessions. The intertrial interval was 4 s. (Breaks were given to the brain-damaged subjects as needed). Each experimental set was preceded by a series of practice trials that included the normal rate sentence frame coupled with each target stimulus. This was done to familiarize listeners with all stimuli along the continua and to ensure that the brain-damaged subjects understood which word in the sentence to make a decision about.

Stimuli were presented by computer over headphones to subjects seated in front of a response board with buttons labeled "b/p" or "d/t." Subjects were instructed to listen to the sentence and decide as quickly and accurately as possible whether the final word in each sentence began with a "b" or a " $p$ " (for the labial series; " $d$ " or " $t$ " in the case of the alveolar series). They were to indicate their decision by pressing the corresponding button on the response board. Responses and reaction times (RTs) were recorded by the computer. Testing was completed individually in a quiet room using closed headphones.

\section{Stimuli and Procedures-Semantic Bias (Experiment 2)}

Stimuli for this experiment were created in an analogous manner to those of Experiment 1. The sentence frames in this case were semantically biased to the voiced ([b]/[p]: She needs hot water for the ___ $;[\mathrm{d}] /[\mathrm{t}]$ : She drives the car with the ) or voiceless endpoint ([b]/[p]: She likes to jog 
along the ; $[\mathrm{d}] /[\mathrm{t}]$ : She saw the show in the

). The continua were identical to those of Experiment 1 but target words were now paired with the semantically biased sentence frames. All other procedures were identical to Experiment 1.

\section{Data Analysis}

Experiment 1: Rate of speech. In order to determine whether the speaking rate of a carrier phrase influenced phonetic identification decisions, both category boundary values and overall percentage voiced responses were computed for each listener for both continua. Category boundaries (computed by fitting a regression line to the identification functions and interpolating the $50 \%$ crossover point, following Miller \& Dexter, 1988) could only be calculated for those individuals who demonstrated some consistency in identification of both endpoints. For the bath-path continuum, 6 of 13 nonfluent aphasic patients were excluded from boundary calculations due to inconsistent responding in one or both rate contexts; 1 of 7 fluent aphasic patients was excluded for similar reasons. For the dent-tent continuum, 5 nonfluent aphasic patients had to be excluded, but no fluent patients were excluded.

Experiment 2: Semantic bias. As in Experiment 1, both category boundaries and overall percentage voiced responses were computed for each listener for both semantically biased continua. Again as in Experiment 1, the results for several individuals in each aphasic group had to be excluded from the boundary calculations due to inconsistency in responses to the endpoint stimuli or across the continua. A total of 9 of 13 nonfluent aphasic patients and 3 of 7 fluent aphasic patients were excluded for the [b]-[p] continuum; 7 of 13 nonfluent aphasics and 1 of 7 fluent aphasic patients were excluded for the $[\mathrm{d}]-[\mathrm{t}]$ continuum.

\section{RESULTS}

\section{Experiment 1: Rate of Speech}

Figure 1 displays the average identification functions along the [b]-[p] continuum for each subject group (for those subjects for whom boundaries were computed). As evident in the figure, all groups exhibited an influence of speaking rate, with a boundary shift of $2.61 \mathrm{~ms}$ for the normal group, $4.33 \mathrm{~ms}$ for the nonfluent aphasic group, and $3.32 \mathrm{~ms}$ for the fluent aphasic group. The boundary values were submitted to a Group $\times$ Rate Context analysis of variance (ANOVA) using log-transformed step values. (The transformation was applied due to unequal group sizes.) Results of the ANOVA revealed a main effect for Rate only $[F(1,22)=17.114, p<.001]$, confirming the rate-related boundary shifts illustrated in Fig. 1. An ANOVA was also conducted on the overall percentage voiced responses across the continuum steps. Somewhat surprisingly, this analysis yielded no main effects or interactions.

Average identification functions along the $[\mathrm{d}]-[\mathrm{t}]$ continuum are displayed in Fig. 2 for each group separately. As may be seen, all groups again demonstrated effects of speaking rate on phonetic identification. The boundary shifts were $3.44 \mathrm{~ms}$ for the normal group, $2.14 \mathrm{~ms}$ for the nonfluent aphasic group, and $2.65 \mathrm{~ms}$ for the fluent aphasic group. A Group $\times$ Rate Context ANOVA on log-transformed boundary values yielded main effects of Group $[F(2,24)=5.156, p<.02]$ and Rate $[F(1,24)=$ $20.795, p<.001]$ but no interaction. As with the [b]-[p] continuum, an ANOVA on the percentage voiced responses across the continuum failed to yield any main effects or interactions.

\section{Experiment 2: Semantic Bias}

Mean identification functions for the labial continuum (for only those subjects for whom boundaries were computed) are presented in Fig. 3 for each subject group. All groups displayed effects of the semantically biased context, with somewhat larger differences shown by both aphasic patient groups relative to the normal controls. In terms of VOT values, the sentence context effect, reflected in the shift in phonetic 


\section{Normal Adults}

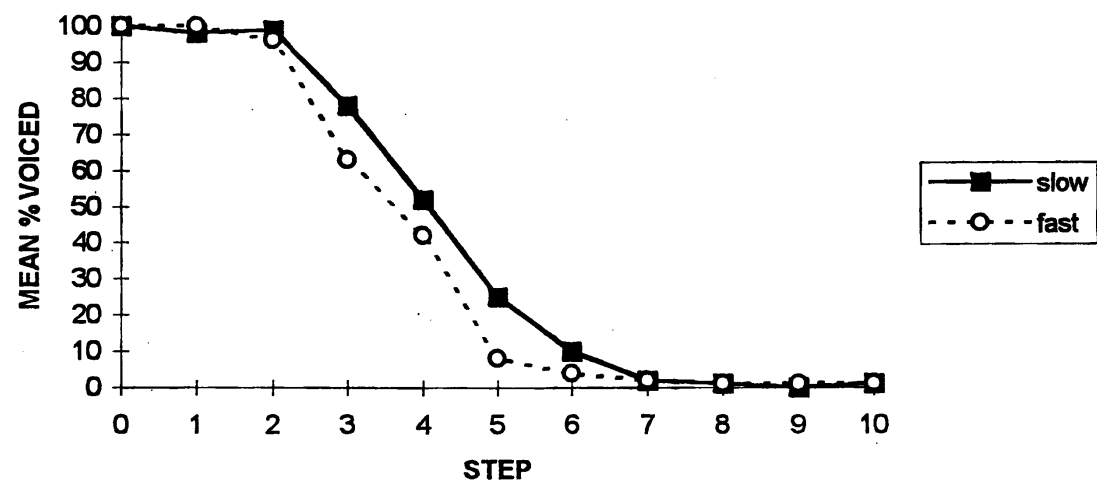

\section{Nonfluent Aphasics}

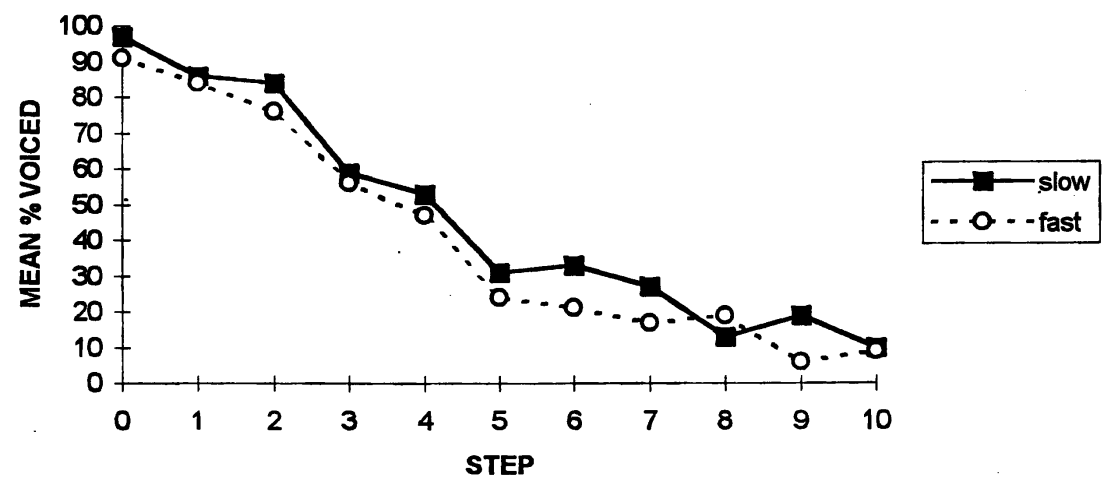

Fluent Aphasics

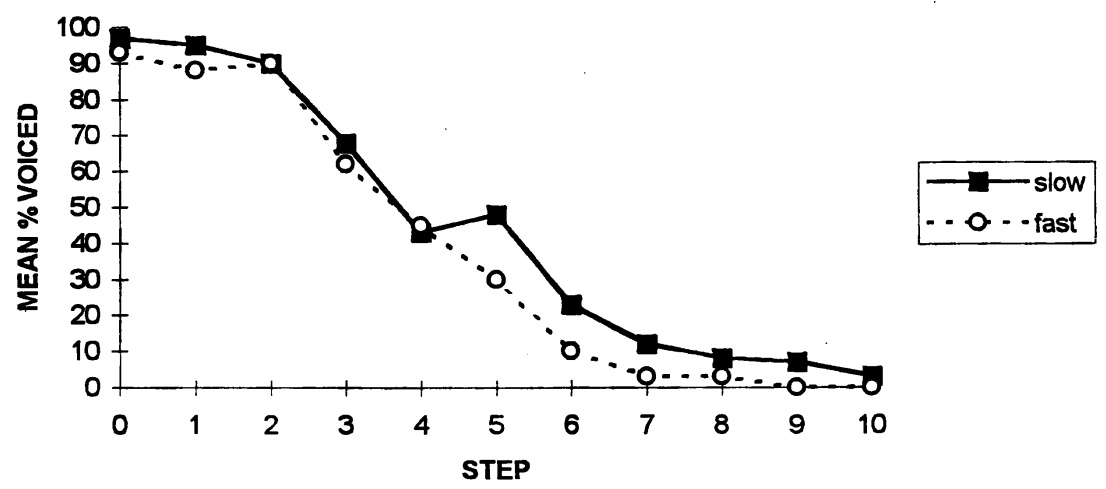

FIG. 1. Mean identification functions for each group for labial rate of speech continua. 


\section{Normal Adults}

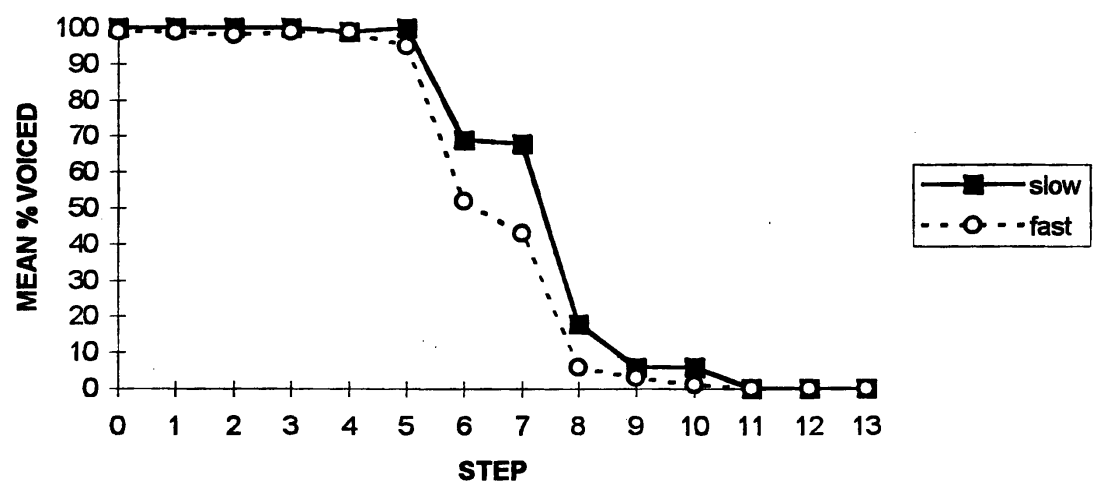

Nonfluent Aphasics

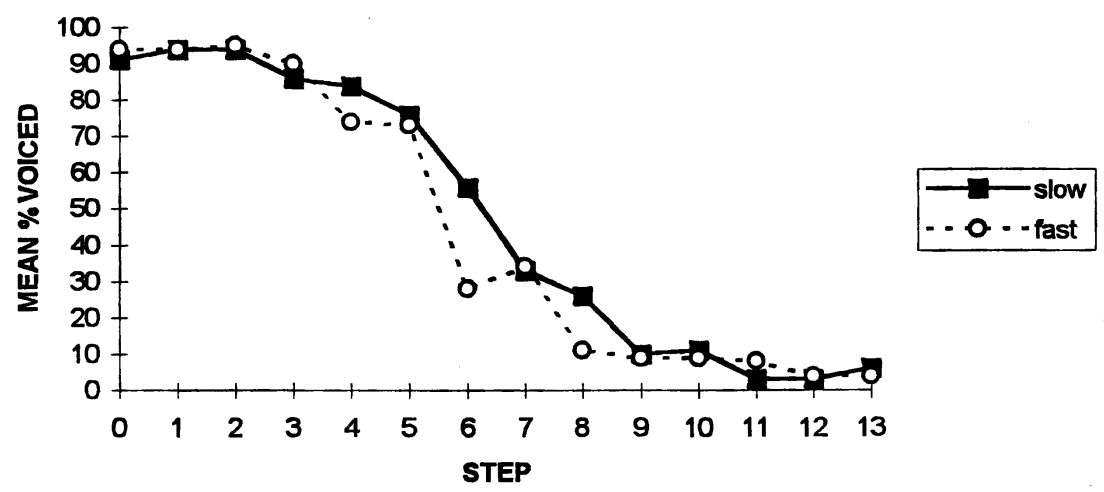

Fluent Aphasics

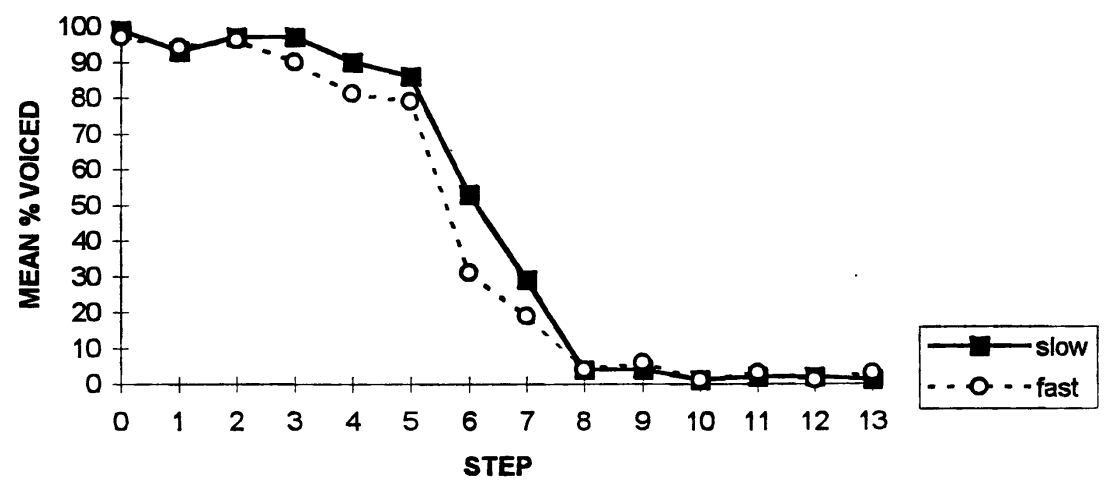

FIG. 2. Mean identification functions for each group for alveolar rate of speech continua. 


\section{Normal Adults}

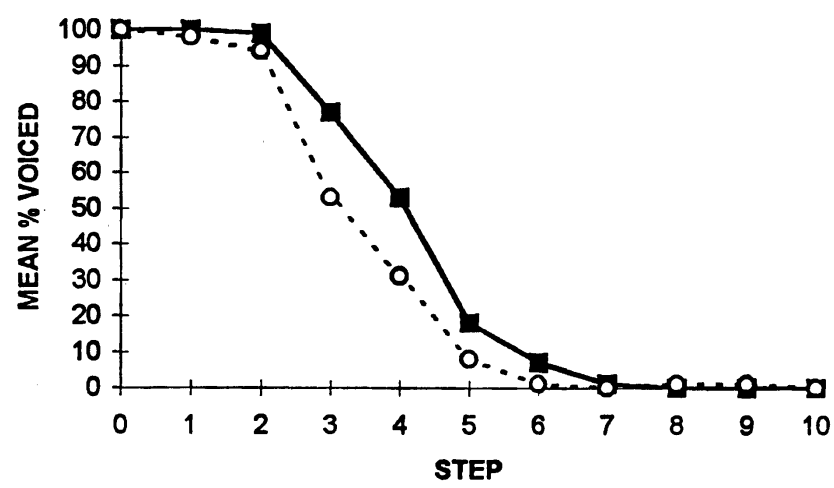

Nonfluent Aphasics

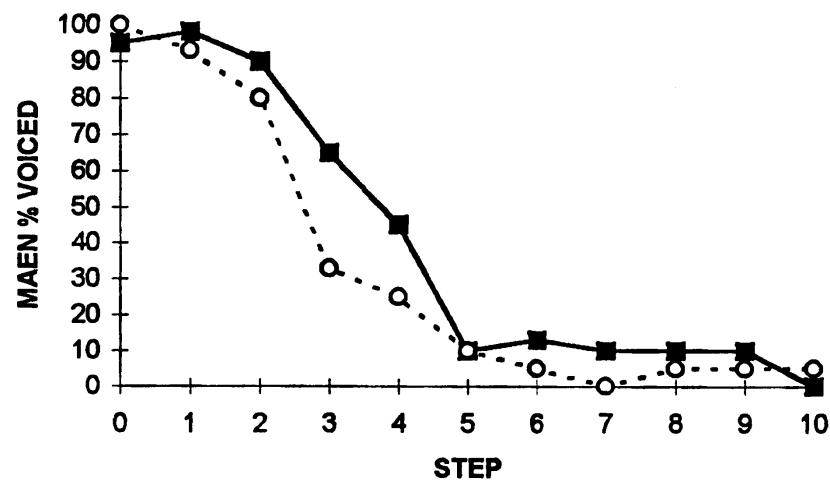

Fluent Aphasics

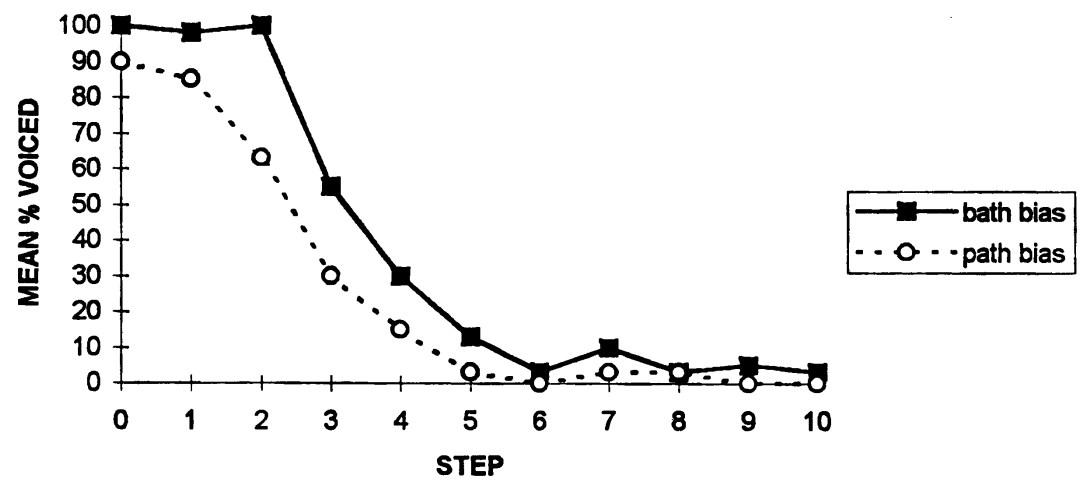

FIG. 3. Mean identification functions for each group for labial semantically biased continua. 


\section{Normal Adults}

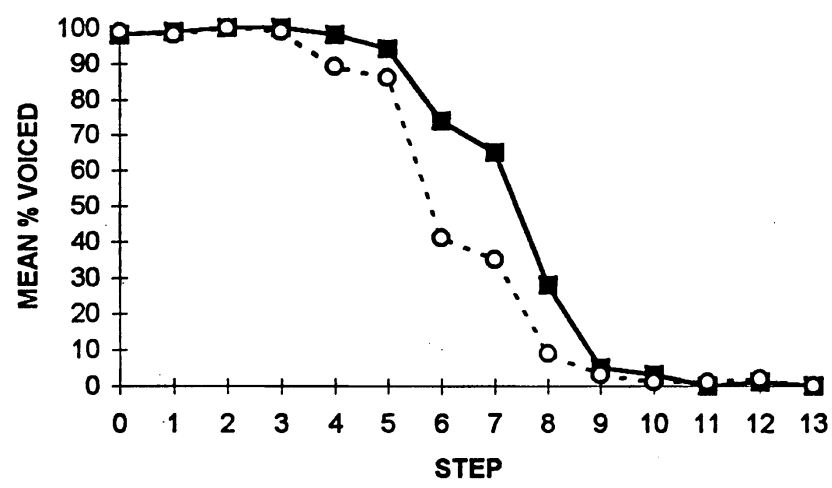

Nonfluent Aphasics

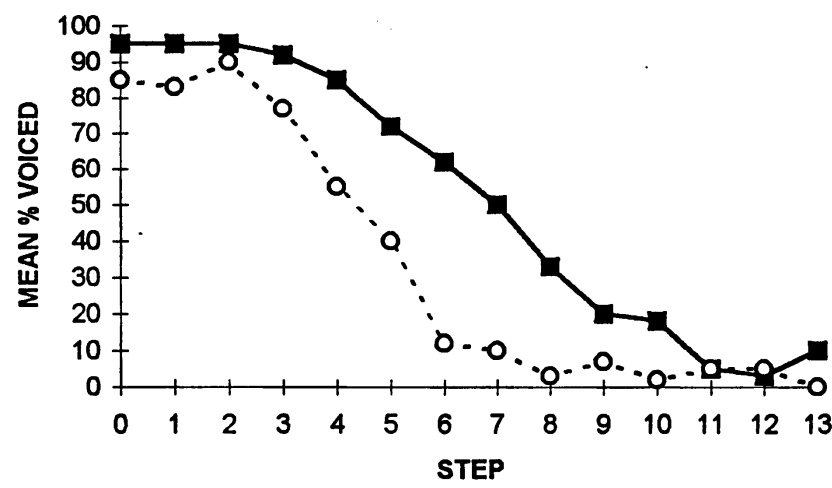

Fluent Aphasics

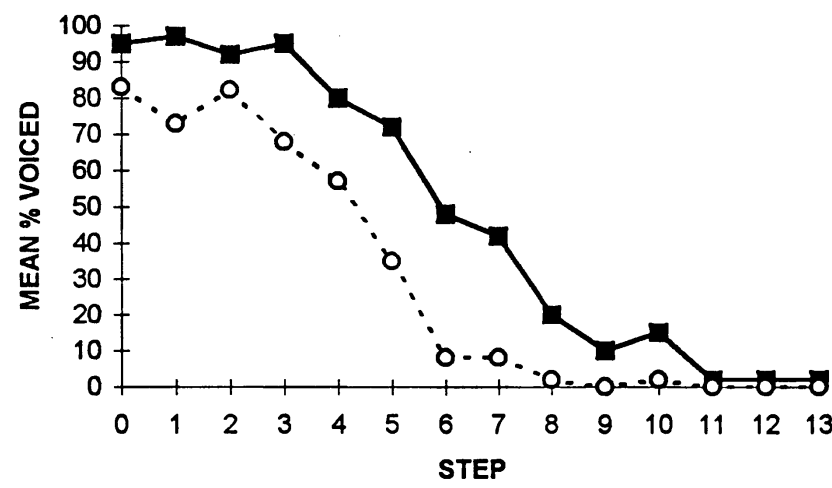

FIG. 4. Mean identification functions for each group for alveolar semantically biased continua. 


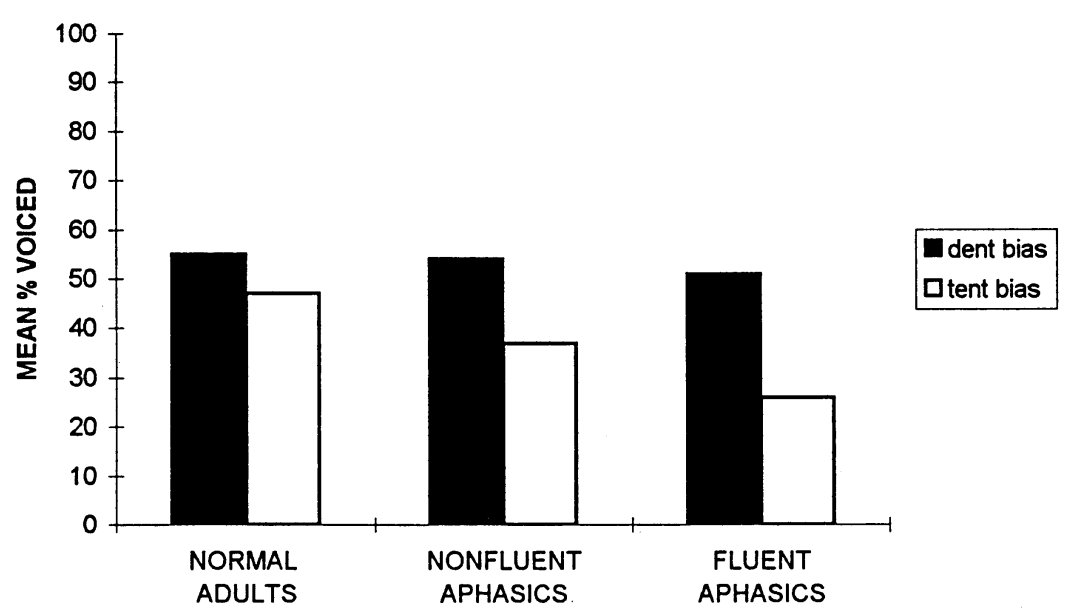

FIG. 5. Mean percentage of voiced responses for each group (all subjects) for alveolar semantically biased continua.

boundaries, was on the order of $3.04 \mathrm{~ms}$ for the normal subjects, whereas it was 4.69 and $5.72 \mathrm{~ms}$ for the nonfluent and fluent aphasic patients, respectively. Logtransformed boundary values were submitted to a Group $\times$ Semantic Bias ANOVA, which yielded a main effect of Semantic Bias $[F(1,17)=27.272, p<.001]$ and a trend toward a Group $\times$ Semantic Bias interaction $[F(2,17)=3.37, p=.06]$. Post hoc analysis of the interaction using the Newman-Keuls procedure $(p<.05)$ demonstrated that only within the fluent aphasic group was the boundary difference significant. An ANOVA conducted on the overall percentage voiced responses yielded only a main effect of Semantic Bias $[F(1,29)=15.327, p<.001]$.

Figure 4 displays the average identification functions for the alveolar continuum for each group. As with the labial continuum, all groups showed the expected boundary shift, again with the normal subjects displaying by far the smallest difference of $3.99 \mathrm{~ms}$ as compared to very large differences of $11.68 \mathrm{~ms}$ for the nonfluent aphasic patients and $11.1 \mathrm{~ms}$ for the fluent aphasic group. A Group $\times$ Semantic Bias ANOVA on $\log$-transformed boundary values yielded main effects of $\operatorname{Group}[F(2,21)=9.9$, $p<.001]$ and Semantic Bias $[F(1,21)=37.558, p<.001]$ as well as a Group $\times$ Semantic Bias interaction $[F(2,21)=4.839, p<.02]$. Post hoc analysis of the interaction using the Newman-Keuls procedure revealed a significant boundary effect for both aphasic patient groups, but no significant difference for the normals.

An ANOVA on the overall percentage of voiced responses yielded main effects of Group $[F(2,29)=3.354, p<.05]$ and Semantic Bias $[F(1,29)=20.770, p<$ $.001]$, but no interaction. The Group effect reflects the much lower percentage voiced responses in the [t]-biased context for the patient groups relative to the normals and the higher percentage voiced responses in the [d]-biased context. These values are plotted in Fig. 5.

\section{DISCUSSION}

One of the major objectives of the present investigation was to determine whether both speaking rate context and semantic context would influence the phonetic identification performance of fluent and nonfluent aphasic patients in a manner comparable to normal listeners. Based on previous findings (e.g., Blumstein et al., 1994), it was predicted that nonfluent aphasic patients would exhibit an increased reliance on se- 
mantic contextual information in rendering phonetic decisions (but cf. Boyczuk \& Baum, 1999); such an increased reliance on context was not necessarily expected for the rate of speech manipulation, due to the temporal processing impairments typically found for nonfluent aphasic patients (e.g., Baum, 1998; Baum et al., 1997). For the fluent aphasic patients, the predicted patterns were different, with a smaller influence of semantic context expected-perhaps even an absence of a contextual bias effect due to deficits in semantic comprehension abilities in this group.

The findings of the two experiments were consistent with some, but not all, of these predictions. In particular, the results of the rate of speech experiment (Experiment 1) revealed an influence of speaking rate on voicing identification for normal control subjects, in keeping with earlier findings (e.g., Miller et al., 1984; Summerfield, 1981). The speaking rate effects were confined to the ambiguous boundary regions of the continua. For those aphasic patients in both clinical groups for whom phonetic boundaries could be computed, a normal speaking rate effect emerged as well, with small but significant boundary shifts of similar magnitude to those of the normal listeners across both labial and alveolar continua. However, as in earlier investigations (e.g., Blumstein et al., 1977, 1984), a relatively large number of patientsnotably in the nonfluent aphasic group-were unable to consistently label even the endpoint stimuli, suggesting either instability in their representations of the phonetic categories or difficulties with the somewhat metalinguistic task of assigning a category label. The responses of these individuals did not, for the most part, indicate a strong influence of rate context, overriding the VOT cues in the stimuli along the continua. Further, for those patients who could consistently label the endpoints, in contrast with our predictions, the temporal processing impairment typical of the nonfluent aphasic patients did not seem to impede their normal perception of speaking rate, as reflected in the normal phonetic boundary shifts.

Perhaps more interesting were the findings for the semantic bias experiment (Experiment 2) in which differences across the subject groups did emerge (although these differences were, again, not entirely consistent with expectations). Of note, first of all, was the finding that more subjects in both aphasic groups had to be excluded from the boundary calculations. Interestingly, in contrast to the results of Experiment 1, many of the exclusions ( 7 of 20 from 2 fluent and 3 nonfluent aphasic patients) were due to an overriding influence of (semantic) context, yielding identification responses entirely consistent with the sentential bias, irrespective of VOT values. Thus, in addition to potentially unstable phonetic categories, the listeners exhibited an overreliance on semantic context; (whether such an overreliance may be strategic is considered below). In addition to more of the exclusions reflecting an influence of semantic context, so too did the phonetic identification functions for those individuals for whom boundaries could be computed. Across both labial and alveolar continua, all groups demonstrated a shift in voicing category boundaries (as shown in Figs. 3 and 4). However, the magnitudes of those shifts were considerably larger for the aphasic patients, particularly on the alveolar continua. In fact, statistically, only the boundary shift for the fluent aphasic patients was significant on the labial continua; on the alveolar continua, both aphasic groups exhibited significant boundary shifts, whereas the small shift demonstrated by the normal control subjects failed to reach significance. Some of the increased semantic context effect in the aphasic patients may be attributable to the slower response times of the brain-damaged patients relative to the normal controls. While reaction time was not a parameter of interest in the current study, overall (and not surprisingly), the patients' response times were substantially longer than those of the normal listeners. The increased response times could have permitted more time for postperceptual context effects to build up. 
For the nonfluent aphasic patients, these results are in keeping with predictions of an increased reliance on context, perhaps as a heuristic strategy to compensate for phonetic processing difficulties, particularly for stimuli containing ambiguous acoustic cues (Blumstein et al., 1994; Boyczuk \& Baum, 1999; see also Caplan \& AydelottUtman, 1994). The large effect of semantic context for the fluent aphasic patients was more surprising. Recall that in Blumstein et al.'s (1994) original investigation of lexical effects on phonetic identification, no lexical influence emerged for their fluent patient group, which was interpreted as reflecting an inability to utilize heuristic strategies in phonetic processing. However, Boyczuk and Baum (1999) reported a relatively normal pattern of lexical effects on phonetic identification in the fluent aphasic patients they studied. Boyczuk and Baum (1999) pointed to differences in the composition of the subject groups and the stimuli used as possible factors contributing to the inconsistent findings. Differences within the patient populations, of course, remain a possibility in reconciling the present results with those of Blumstein and colleagues (1994). In particular, the fluent aphasic patients in the current investigation included more individuals who would be classified as having evolved to anomic aphasia; thus the patients may represent a less severely involved (or recovered) group. The stimuli differed across the experiments in several ways as well. The stimuli in the present investigation may be considered somewhat less natural than those used by Blumstein and colleagues (1994) due to the fact that amplitude of the burst and aspiration noise were not manipulated (Burton et al., 1989). Another interesting difference across the studies is that both labial and alveolar stimuli were used in the present study, whereas only alveolar stops were examined in Blumstein et al. (1994). It is noteworthy that, in the present experiments, larger contextual influences emerged for the alveolar continua relative to the labial continua. Pitt and Samuel (1993) have remarked that the greatest variability in the emergence of lexical influences on phonetic identification occurs for alveolar voicing contrasts. That is, the $[\mathrm{d}]-[\mathrm{t}]$ contrast appears to yield the most fragile effects of lexical status, failing to emerge in some instances (e.g., Burton et al., 1989), whereas labial and velar voicing contrasts more consistently show lexical effects (Pitt \& Samuel, 1993). While the present investigation did not examine a lexical-level influence in particular, it does not seem unreasonable to have expected a similar fragility in higher level context effects for the alveolar voicing contrast. In fact, the opposing pattern that emerged in the present investigation (i.e., larger context effects for alveolar relative to labial stimuli) suggests a reevaluation of the explanations for the absence of context effects in speech perception (e.g., Pitt \& Samuel, 1993) may be in order. One must hasten to note, however, that while the normal subjects displayed a somewhat larger context effect on the alveolar continua relative to the labial continua, it was the brain-damaged patients who showed a dramatic difference across the places of articulation; therefore, any conclusions regarding normal speech perception processes must be tempered to some degree.

Although no direct comparisons were made across the two experiments, it is interesting to consider why certain differential patterns emerged. As noted in the introduction, the two contextual influences examined - rate of speech and semantic biasreflect the potential influence of different levels of processing and possibly different processing mechanisms or routines. That is, the effect of speaking rate, although signaled in the sentence context, is still an effect of acoustic-phonetic structure and, as such, may occur at a relatively low level in the speech processing system. Moreover, perceptual adjustments to alterations in speaking rate may be automatically invoked under conditions of ambiguity (Miller et al., 1984) and may not reflect postperceptual processing. The automaticity under conditions of uncertainty may explain why the speaking rate effects emerged only when examining category boundary values and 
not overall percentage voiced responses across the continua. In contrast, the influence of semantic context emerges from a higher level of language comprehension and is likely to reflect conscious, strategic processing to a greater extent (see Borsky et al., 1998; Connine, 1987; Miller et al., 1984 for various interpretations). The semantic contextual bias may influence phonetic identification responses across the continua, not solely in the ambiguous stimuli region, due to the adoption of a particular strategic mode of processing. An alternative possibility is simply that the semantic effects are stronger due to natural sentence interpretation processes that cannot be ignored.

To return to the main goals of the study, in sum, the findings of the current set of experiments revealed that individual nonfluent and fluent aphasic patients exhibited impairments in phonetic perception (Blumstein et al., 1977, 1984) and that both groups of patients in the present study may have relied to a greater extent than normal individuals on semantic context to compensate, in part, for such perceptual deficits (see also Boyczuk \& Baum, 1999). Somewhat surprisingly, the nonfluent aphasic patients displayed normal effects of speaking-rate modifications on phonetic identification, suggesting that any temporal processing deficits they exhibit may be restricted to certain aspects of speech perception. Alternatively, perhaps the difficulty that nonfluent aphasics demonstrate in perceiving temporal cues in speech represents a qualitative rather than a quantitative difference from normals. In other words, perhaps such patients often require more salient cues (i.e., longer durational differences across segments or phrases; see, e.g., Tallal \& Newcombe, 1978; but cf. Blumstein, Tartter, Nigro, \& Statlender, 1984; Riedel \& Studdert-Kennedy, 1985) to adequately process temporal attributes of speech. In the present investigation, the speaking rate alterations appear to have been large enough to be perceptible to the patients. The results of the present study do not support a clear dissociation between the fluent and nonfluent patient groups in terms of their reliance on strategic heuristics in phonetic identification (cf. Blumstein et al., 1994; Boyczuk \& Baum, 1999). Nonetheless, the available data remain limited and additional research must be completed prior to reaching more definitive conclusions.

\section{REFERENCES}

Baum, S. (1998). The role of fundamental frequency and duration in the perception of linguistic stress by individuals with brain damage. Journal of Speech, Language, \& Hearing Research, 41, 31-40.

Baum, S., Pell, M., Leonard, C., \& Gordon, J. (1997). The ability of right- and left-hemisphere-damaged individuals to produce and interpret prosodic cues marking phrasal boundaries. Language \& Speech, 40, 313-330.

Blumstein, S. (1991). Phonological aspects of aphasia. In Sarno (Ed.), Acquired aphasia. San Diego, CA: Academic Press.

Blumstein, S., Milberg, W., \& Shrier, R. (1982). Semantic processing in aphasia: Evidence from an auditory lexical decision task. Brain \& Language, 17, 301-315.

Blumstein, S. E., Burton, M., Baum, S., Waldstein, R., \& Katz, D. (1994). The role of lexical status on the phonetic categorization of speech in aphasia. Brain and Language, 46, 181-197.

Blumstein, S. , Tartter, V., Nigro, G., \& Statlender, S. (1984). Acoustic cues for the perception of place of articulation in aphasia. Brain and Language, 22, 128-149.

Borsky, S., Tuller, B., \& Shapiro, L. (1998). "How to milk a coat": The effects of semantic and acoustic information on phoneme categorization. Journal of the Acoustical Society of America, 103, 26702676.

Boyczuk, J., \& Baum, S. (1999). The influence of neighborhood density on phonetic categorization in aphasia. Brain and Language, 67, 46-70.

Burton, M. W., Baum, S. R., \& Blumstein, S. E. (1989). Lexical effects on the phonetic categorization of speech: The role of acoustic structure. Journal of Experimental Psychology, 15(3), 567-575.

Caplan, D. (1992). Language: Structure, process, and disorders. Cambridge, MA: MIT Press. 
Caplan, D., \& Aydelott-Utman, J. (1994). Selective acoustic-phonetic impairment and lexical access in an aphasic patient. Journal of the Acoustical Society of America, 95, 512-517.

Connine, C. (1987). Constraints on interactive processes in auditory word recognition: The role of sentence context. Journal of Memory and Language, 26, 527-538.

Connine, C., Blasko, D., \& Hall, M. (1991). Effects of subsequent sentence context in auditory word recognition: Temporal and linguistic constraints. Journal of Memory \& Language, 30, 234-250.

Elman, J. L., \& McClelland, J. L. (1986). Exploiting lawful variability in the speech wave. In J. S. Perkell \& D. H. Klatt (Eds.), Invariance and variability in speech processes (pp. 360-381). Hillsdale, NJ: Erlbaum.

Fodor, J. (1983). The modularity of mind. Cambridge, MA: MIT Press.

Fox, R. A. (1984). Effect of lexical status on phonetic categorization. Journal of Experimental Psychology: Human Perception and Performance, 10(4), 526-540.

Ganong, W. F. (1980). Phonetic categorization in auditory word perception. Journal of Experimental Psychology: Human Perception and Performance, 6(1), 110-125.

Goodglass, H., \& Kaplan, E. (1983). The assessment of aphasia and related disorders. Philadelphia, PA: Lea \& Febiger.

Gow, D., \& Caplan, D. (1996). An examination of impaired acoustic-phonetic processing in aphasia. Brain and Language, 52, 386-407.

Leonard, C., \& Baum, S. (1997). The influence of phonological and orthographic information on auditory lexical access in brain-damaged patients: A preliminary investigation. Aphasiology, 11, 1031-1041.

Luce, P. (1987). Neighborhoods of words in the mental lexicon. Unpublished doctoral dissertation, Indiana University, Bloomington, IN.

McClelland, J., \& Elman, J. (1986). The TRACE model of speech perception. Cognitive Psychology, 18, $1-86$.

McQueen, J. (1991). The influence of the lexicon on phonetic categorization: Stimulus quality in wordfinal ambiguity. Journal of Experimental Psychology: Human Perception and Performance, 17, $433-443$.

Mertus, J. (1989). BLISS User's Manual. Providence, RI: Brown University Press.

Milberg, W., Blumstein, S., \& Dworetzky, B. (1988). Phonological factors in lexical access: Evidence from an auditory lexical decision task. Bulletin of the Psychonomic Society, 26(4), 305-308.

Miller, J. L., \& Dexter, E. R. (1988). Effects of speaking rate and lexical status on phonetic perception. Journal of Experimental Psychology: Human Perception and Performance, 14, 369-378.

Miller, J. L., Dexter, E. R., \& Pickard, K. A. (1984). Influence of speaking rate and lexical status on word identification. Paper presented at the Acoustical Society of America, Minnesota.

Newman, R., Sawusch, J., \& Luce, P. (1997). Lexical neighborhood effects in phonetic processing. Journal of Experimental Psychology: Human Perception and Performance, 23, 873-889.

Pitt, M., \& Samuel, A. (1993). An empirical and meta-analytic evaluation of the phoneme identification task. Journal of Experimental Psychology: Human Perception and Performance, 19, 699-725.

Riedel, K., \& Studdert-Kennedy, M. (1985). Extending formant transitions may not improve aphasics' perception of stop consonant place of articulation. Brain and Language, 24, 223-232.

Samuel, A. (1981). Phonemic restoration: Insights from a new methodology. Journal of Experimental Psychology: General, 110, 474-494.

Summerfield, Q. (1981). Articulatory rate and perceptual constancy in phonetic perception. Journal of Experimental Psychology: Human Perception and Performance, 7, 1074-1095.

Tallal, P., \& Newcombe, F. (1978). Impairment of auditory perception and language comprehension in dysphasia. Brain and Language, 5, 13-24.

Warren, R. (1970). Perceptual restoration of missing speech sounds. Science, 167, 392-395. 